Research Paper

\title{
Enhancing inulinase yield by irradiation mutation associated with optimization of culture conditions
}

\author{
Yafeng Gou, Jianhua Li, Junbao Zhu, Wanyun Xu, Jianfeng Gao \\ College of Life Science, Shihezi University, Xinjiang, China.
}

Submitted: November 3, 2014; Approved: January 27, 2015.

\begin{abstract}
A new inulinase-producing strain was isolated from rhizosphere soils of Jerusalem artichoke collected from Shihezi (Xinjiang, China) using Jerusalem artichoke power (JAP) as sole carbon source. It was identified as an Aspergillus niger strain by analysis of $16 \mathrm{~S}$ rRNA. To improve inulinase production, this fungus was subjected to mutagenesis induced by ${ }^{60} \mathrm{Co} \gamma$-irradiation. A genetically stable mutant (designated E12) was obtained and it showed 2.7-fold higher inulinase activity $(128 \mathrm{U} / \mathrm{mL})$ than the parental strain in the supernatant of a submerged culture. Sequential methodology was used to optimize the inulinase production of stain E12. A screening trial was first performed using Plackett-Burman design and variables with statistically significant effects on inulinase bio-production were identified. These significant factors were further optimized by central composite design experiments and response surface methodology. Finally, it was found that the maximum inulinase production $(185 \mathrm{U} / \mathrm{mL})$ could be achieved under the optimized conditions namely $\mathrm{pH} 7.0$, yeast extract concentration of $5.0 \mathrm{~g} / \mathrm{L}$, JAP concentration of $66.5 \mathrm{~g} / \mathrm{L}$, peptone concentration of $29.1 \mathrm{~g} / \mathrm{L}$, solution volume of $49.4 \mathrm{~mL}$ in $250-\mathrm{mL}$ shake flasks, agitation speed of $180 \mathrm{rpm}$, and fermentation time of $60 \mathrm{~h}$. The yield of inulinase under optimized culture conditions was approximately 1.4-fold of that obtained by using basal culture medium. These findings are of significance for the potential industrial application of the mutant E12.
\end{abstract}

Key words: inulin, ethanol fermentation, inulinase, central composite design, response surface methodology.

\section{Introduction}

Jerusalem artichoke (Helianthus tuberosus L.) is a native plant of North America and its tubers contain about $75-85 \%$ (dry weight) of total sugars (Wang et al., 2013). As an excellent source of sugar and renewable raw material, Jerusalem artichoke grows well in poor soil and even in sand, and has a high tolerance to frost and various plant diseases. Inulin is the primary storage carbohydrate of Jerusalem artichoke. As a natural carbohydrate, inulin occurs in plants mainly as an energy reserve and as a cryoprotectant with beneficial nutritional and technological properties (Saengthongpinit and Sajjaanantakul, 2005). Inulin has a polymerization degree (DP) 2-60 or higher, consisting of linear $\beta$-2, 1-linked polyfructose chains displaying a terminal glucose unit. Recently, the production of ethanol by fer- mentation of Jerusalem artichoke tubers has become a hot research issue (Yuan et al., 2008; Zhang et al., 2010; Hu et al., 2012; Li et al., 2013).

Recently, inulinases have received much attention as they can be widely applied to hydrolyze inulin for the production of fuel ethanol, fructose, and fructo-oligosaccharides (Gao et al., 2009). Inulinases are a group of hydrolases which target on the $\beta-2,1$ linkage of inulin and hydrolyze it into fructose and glucose. Inulinases can be divided into endo-inulinase and exo-inulinase. The endo-inulinase hydrolyzes the internal linkages in inulin to produce inulotriose, inulotetraose, and inulopentaose as the main products. The exo-inulinase hydrolyzes inulin into fructose and glucose, then, the formed fructose and glucose can be further fermented into ethanol by specific microorganisms ( $\mathrm{Li}$ et al., 2013).

Send correspondence to J. Gao. College of Life Science, Shihezi University, Shihezi 832003 Xinjiang, China. E-mail: jianfenggao@126.com, jianfengg@shzu.edu.cn. 
Inulinases can be secreted by a variety of microbes including fungi, yeast, and bacteria. Among them, Aspergillus and Kluyveromyces strains are generally preferred choices for commercial applications (Zhang et al., 2004). Recently, many studies have been conducted using inulinase from Aspergillus for enzymatic hydrolysis of inulin (Gill et al., 2006; Sirisansaneeyakul et al., 2006). Some efforts have been made to enhance enzyme activity of Aspergillus such as transgene expression (Zhang et al., 2004) and coculture with other species (Ge et al., 2009).

Irradiation mutagenesis by means of X-rays, ultraviolet rays, and $\gamma$-rays can cause morphological and biochemical alterations in microbial cells. Feng et al. (2014) found that nuclear irradiation could remarkably enhance the lipid yields of Nitzschia sp. Cheng et al. (2014) reported that the biomass and lipid yields of Nitzschia sp. were increased by $53.8 \%$ and $28.1 \%$, respectively, through ${ }^{60} \mathrm{Co}-\gamma$-ray-induced mutation at $900 \mathrm{~Gy}$. However, research on the utilization of nuclear irradiation to enhance the inulinase activity of microbes is very scarce.

Thereupon, the objectives of the present study were (1) to isolate an inulinase-producing strain and re-mutate it by using ${ }^{60} \mathrm{Co}-\gamma$ radiation; (2) to optimize the conditions for inulinase production by the mutant.

\section{Materials and Methods}

\section{Chemicals}

Jerusalem artichoke dry powder was purchased from Xi'an Sinuote Biotechnology Co., Ltd., China. The Jerusalem artichoke powder (JAP) was used as carbon source for microbial screening, fermentation and inoculation. The JAP contains (w/w): $2.8 \%$ fructose, $3.2 \%$ sucrose, $80.7 \%$ inulin, $1.4 \%$ nitrogen, $4.9 \%$ crude fiber, $4.3 \%$ ash, and $2.7 \%$ water. Yeast extract and peptone, with nitrogen contents of $9.8 \%$ and $12.2 \%(\mathrm{w} / \mathrm{w})$ respectively, were purchased from Aoboxing Biotechnology Co., Ltd. (Beijing, China). Inulin ( $\geq 98 \%$ purity) was obtained from SigmaAdrich (St Louis, MO, USA). All other chemicals were of analytical grade and commercially available.

\section{Isolation of inulinase-producing fungus strain}

The rhizosphere soils of Jerusalem artichoke collecting from Shihezi (Xinjiang, China) were chosen as natural isolation sources of microbes. Enrichment of microorganisms was carried out from $5 \mathrm{~g}$ of individual soil samples with $50 \mathrm{~mL}$ of selecting medium (JAP $40.0 \mathrm{~g} / \mathrm{L}, \mathrm{Na}_{2} \mathrm{HPO}_{4}$ $\left.10.0 \mathrm{~g} / \mathrm{L}, \mathrm{NH}_{4} \mathrm{Cl} 20.0 \mathrm{~g} / \mathrm{L}, \mathrm{pH} 7.0\right)$ in a $250-\mathrm{mL}$ flask in a 28 ${ }^{\circ} \mathrm{C}$ incubator (180 rpm) for 4 days. Then an aliquot of culture medium was streaked onto selecting agar medium (selecting medium with 2\% agar) and incubated for 3-5 days at $28{ }^{\circ} \mathrm{C}$. After incubation, all the plates were observed and morphologically different colonies (fungus-like) were selected and purified by streaking over the same medium. For purification, each isolate was transferred at least three times. For further screening and identification, the isolates were maintained on Czapek yeast agar (CYA) medium $\left(\mathrm{K}_{2} \mathrm{HPO}_{4} 1.0 \mathrm{~g} / \mathrm{L}\right.$, yeast extract $5.0 \mathrm{~g} / \mathrm{L}$, sucrose $30.0 \mathrm{~g} / \mathrm{L}$, agar $15.0 \mathrm{~g} / \mathrm{L}$, Czapek concentrate $10.0 \mathrm{~mL} / \mathrm{L}$, distilled water $1000 \mathrm{~mL}$ ) (Silva et al., 2011). The composition of Czapek concentrate is $(\mathrm{g} / \mathrm{L}): \mathrm{NaNO}_{3}$ 300.0, $\mathrm{KCl} 50.0$, $\mathrm{FeSO}_{4} 7 \mathrm{H}_{2} \mathrm{O} 1.0, \mathrm{ZnSO}_{4} 7 \mathrm{H}_{2} \mathrm{O} 1.0, \mathrm{CuSO}_{4} 5 \mathrm{H}_{2} \mathrm{O} 0.5$.

The isolates showing moderate to excellent activity were selected for secondary screening, which was performed by the spore cultivation method. For this reason, the isolates were transferred to potato dextrose agar (PDA) plates (peeled and sliced potato $200.0 \mathrm{~g} / \mathrm{L}$, glucose 20.0 $\mathrm{g} / \mathrm{L}$, agar $20.0 \mathrm{~g} / \mathrm{L}$ ). Following growth for $3-7$ days at $28^{\circ} \mathrm{C}$, the mycelium was collected and mixed with sterile water (containing $0.05 \%(\mathrm{w} / \mathrm{v})$ Tween 80$)$ to prepare spore suspension (about $10^{5}$ conidia per $\mathrm{mL}$ ). After this, $1 \mathrm{~mL}$ of each conidial suspension was inoculated in a $250-\mathrm{mL}$ shake flask containing $100 \mathrm{~mL}$ of fermentation medium (JAP $40.0 \mathrm{~g} / \mathrm{L}$, yeast extract $5.0 \mathrm{~g} / \mathrm{L}$, peptone $10.0 \mathrm{~g} / \mathrm{L}, \mathrm{pH} 7.0)$. The flasks were incubated in a rotary shaker $(180 \mathrm{rpm})$ at 28 ${ }^{\circ} \mathrm{C}$ for 3-5 days, and then the microorganisms were re-isolated by serial dilution of the culture and plated on the selecting agar medium by adopting standard spread plate method. In this study, all media were autoclaved at $121^{\circ} \mathrm{C}$ for $15 \mathrm{~min}$.

\section{Species identification}

The preferred isolates were transferred to CYA plates. Each plate was inoculated with three $5-\mu \mathrm{L}$ drops of freshly prepared conidial suspension, incubated at $28^{\circ} \mathrm{C}$ for 7 days and observed for species identification by macroscopic and microscopic morphological characteristics as described by Maren (2002). The isolates were also sent to Shanghai Sangon Biotechnology Co., Ltd. (Shanghai, China) for molecular identification. Molecular identification was done by partial sequencing. Genomic DNA was isolated and about $500 \mathrm{bp}$ rDNA fragments were amplified using primers CF1L (5'-GCCGACTCTTTGACYGAR GAR-3') and CF4 (5'-TTTYTGCATCATRAGYTGG AC-3') (Peterson, 2008). Sequencing PCR was done with the BigDye ${ }^{\circledR}$ Terminator v3.1 Cycle Sequencing Kit (ABI, Foster City, CA, USA). Sequence data was aligned with Lasergene sequence analysis software (DNASTAR, Inc., Madison, WI, USA) and homologies to sequences of reference strains were determined by blast search.

\section{Determination of inulinase activity}

The isolates were inoculated into $250-\mathrm{mL}$ shake flasks, each containing $50 \mathrm{~mL}$ of fermentation medium, and then cultured at $28{ }^{\circ} \mathrm{C}$ and $180 \mathrm{rpm}$ for $60 \mathrm{~h}$ on a rotary shaker. Afterwards, the fermented liquid broth was centrifuged at $12000 \mathrm{rpm}$ and $4{ }^{\circ} \mathrm{C}$ for $10 \mathrm{~min}$, and the supernatant was used as the crude enzyme preparation.

The inulinase activity was assayed by measuring the amount of reducing sugar released from inulin using a mod- 
ified 3,5-di nitro salicylic acid (DNS) method (Sheng et al., 2007). In brief, $1.0 \mathrm{~mL}$ of suitably diluted crude enzyme solution was mixed with $4.0 \mathrm{~mL}$ of acetate buffer $(0.1 \mathrm{M}, \mathrm{pH}$ $5.0)$ containing $1.5 \%(\mathrm{w} / \mathrm{v})$ inulin. After incubation at $55^{\circ} \mathrm{C}$ for $10 \mathrm{~min}$ in a water bath, each reaction mixture was mixed with $5.0 \mathrm{~mL}$ of DNS reagent and then kept in boiling water for $5 \mathrm{~min}$. The same mixtures but with inactivated enzyme crude extracts (heated at $100{ }^{\circ} \mathrm{C}$ for $10 \mathrm{~min}$ ) were used as blank controls. After quick cooling on ice, the reaction mixture was assayed for reducing sugars at $540 \mathrm{~nm}$ using a UV-2100 UV/VIS spectrophotometer (UNICO, Shanghai, China). The calibrating curve was drawn for DNS using fructose. One unit of inulinase activity (U) was defined as the amount of enzyme responsible for the production of $1 \mathrm{mmol}$ of reducing sugar per minute under the above conditions. All enzymatic reactions were performed in triplicates.

\section{Mutagenesis}

The cells of isolate exhibiting the highest inulinase activity were mutated by nuclear irradiation. For this, the mycelium was harvested and suspended in sterile water. Five $\mathrm{mL}$ of the suspension in aliquots was transferred to sterile tubes and irradiated at room temperature using a ${ }^{60} \mathrm{Co} \gamma$-ray irradiator, with dosages of 800,1000 and 1200 Gy respectively (92-99\% kill rate). Five $\mu \mathrm{L}$ of each conidial suspension were inoculated to the solid fermentation culture medium for single cell separation after irradiation. After incubation at $28^{\circ} \mathrm{C}$ for $48 \mathrm{~h}$, formed individual colonies were then picked up into 96 micro-well-plates with $0.2 \mathrm{~mL}$ of sterile water. Afterwards, the cell suspension was inoculated to the solid selecting culture medium, incubated at $28^{\circ} \mathrm{C}$ for $36 \mathrm{~h}$. The mutants were grouped to three categories (forward mutation, negative mutation and non-mutation) on the basis of colony diameters. The inulinase activity of mutants was measured as described above.

\section{Plackett-Burman experimental design}

Factors including JAP concentration, yeast extract concentration, peptone concentration, duration, temperature, solution volume, inoculation size and $\mathrm{pH}$ were examined for the fermentation production of inulinase in 250-mL Erlenmeyer flasks. The level of each factor was first estimated through single factor experiments. All experiments were carried out in triplicate and the averages of inulinase activity were taken as the response. The fermentation time was $60 \mathrm{~h}$.

An initial screening test was conducted on all eight components of the medium and culture conditions using Plackett-Burman experimental design. Each variable was defined at two levels, namely a low level (coded by -1) and a high level (coded by 1 ) as listed in Table 1 . The three dummy variables $\left(\mathrm{X}_{4}, \mathrm{X}_{8}\right.$ and $\left.\mathrm{X}_{11}\right)$ were adopted to investigate the experimental errors. The results of PlackettBurman experimental design were fitted by the first-order model as follows (Reddy et al., 2008):

$$
Y=\beta_{0}+\sum \beta_{i} X_{i}(i=1,2, \ldots k)
$$

where $Y$ is the estimated target function, $\beta_{0}$ the model intercept, $\beta_{i}$ the regression coefficient, $X_{i}$ the coded independent factor.

\section{Path of steepest ascent method}

Based on the results of Plackett-Burman experimental design, the optimum level scope of each selected factor was examined by path of steepest ascent method. A path of steepest ascent method was designed using the direction of Plackett-Burman experimental value as the uphill direction. The changing step size was confirmed according to the effect value of Plackett-Burman design. High value was selected when the effect value of variable was positive, and low value was selected when the effect value of variable was negative.

Table 1 - The Plackett-Burman design for screening the variables, and the statistical analysis of variables.

\begin{tabular}{llcccc}
\hline Factor & Variable & Low level (-1) & High level (1) & F value & p value \\
\hline $\mathrm{JAP}(\mathrm{g} / \mathrm{L})$ & $\mathrm{X}_{1}$ & 40.0 & 60.0 & 23.24 & $0.0170^{*}$ \\
$\mathrm{pH}$ & $\mathrm{X}_{2}$ & 5.0 & 7.0 & 0.65 & 0.4790 \\
Yeast extract $(\mathrm{g} / \mathrm{L})$ & $\mathrm{X}_{3}$ & 5.0 & 7.5 & 4.78 & 0.1168 \\
Fermentation time $(\mathrm{h})$ & $\mathrm{X}_{5}$ & 60 & 96 & 0.33 & 0.6080 \\
Peptone $(\mathrm{g} / \mathrm{L})$ & $\mathrm{X}_{6}$ & 10.0 & 15.0 & 50.43 & $0.0057^{* *}$ \\
Temperature $\left({ }^{\circ} \mathrm{C}\right)$ & $\mathrm{X}_{7}$ & 27 & 29 & 0.48 & 0.5385 \\
Solution volume $(\mathrm{mL})$ & $\mathrm{X}_{9}$ & 40 & 60 & 25.97 & $0.0146^{*}$ \\
Inoculation size $(\%)$ & $\mathrm{X}_{10}$ & 4 & 6 & 0.14 & 0.7352 \\
Dummy variable & $\mathrm{X}_{4}, \mathrm{X}_{8}, \mathrm{X}_{11}$ & -1 & 1 & - & - \\
- & Model & - & - & 13.25 & $0.0285^{*}$ \\
\hline
\end{tabular}

$*$ Significant at $95 \%$ confidence degree $(\mathrm{p}<0.05) .{ }^{* *}$ Extremely significant level at $99 \%$ confidence degree $(\mathrm{p}<0.01)$. 


\section{Central composite design}

A central composite design with three variables (Table 2) was used to evaluate the response pattern and to determine the optimum combination of JAP content, peptone content and solution volume for maximizing inulinase production (Plackett-Burman design had shown significant curvature and confirmed the significance of all four parameters). The variables with that were predicted to produce highest inulinase activity by the path of steepest ascent method were used as center-point for the central composite design. Experimental results were fitted to a predictive quadratic polynomial equation as the correlation between the response variable and the independent variables (Xie et al., 2014):

$$
\begin{aligned}
& Y=\beta_{0}+\beta_{1} X_{1}+\beta_{2} X_{2}+\beta_{3} X_{3}+\beta_{11} X_{1}^{2}+ \\
& \beta_{22} X_{2}^{2}+\beta_{33} X_{3}^{2}+\beta_{12} X_{1} X_{2}+\beta_{13} X_{1} X_{3}+ \\
& \beta_{23} X_{2} X_{3}
\end{aligned}
$$

where $Y$ is the predicted response; $\beta_{0}$, intercept; $\beta_{1}, \beta_{2}, \beta_{3}$, linear coefficients; $\beta_{11}, \beta_{22}, \beta_{33}$, quadratic coefficients; and $\beta_{12}, \beta_{13}, \beta_{23}$ are interactive coefficients. The experimental design was developed using Design Expert 8.0.7.1 (Statease, Inc., Minneapolis, MS, USA).

\section{Results and discussion}

\section{Isolation and identification of the Aspergillus}

After the isolation period, a total of 12 inulinaseproducing isolates were recovered from 42 soil samples. These strains could solubilize inulin particles in the agar plates and form clear surrounding zones. This was further confirmed by growing the isolates in liquid medium containing inulin as sole carbon source. Among the 12 isolates tested, one gave maximum enzyme activity $(34.4 \mathrm{U} / \mathrm{mL})$ and it was designated as G-60. The partial 1400 bp $16 \mathrm{~S}$ rRNA gene sequence of the G-60 isolate exhibited 99.3\% homology with Aspergillus niger strain AF10 (Gen-Bank number AF369388). Thus the strain was identified as $A$. niger G-60.

\section{Screening single-celled mutants with high inulinase productivity}

Compared with the limited penetration effect of ultraviolet rays, $\gamma$-rays can generate free radicals, which may al-

Table 2 - Range of variation of the independent factors used in the central composite design.

\begin{tabular}{lcccccc}
\hline Variable & Code & \multicolumn{5}{c}{ Level of variation } \\
\cline { 3 - 7 } & & -1.68 & -1 & 0 & 1 & 1.68 \\
\hline JAP $(\mathrm{g} / \mathrm{L})$ & A & 53.2 & 60.0 & 70.0 & 80.0 & 86.8 \\
Peptone $(\mathrm{g} / \mathrm{L})$ & B & 9.9 & 15.0 & 22.5 & 30.0 & 35.1 \\
Solution volume $(\mathrm{mL})$ & $\mathrm{C}$ & 33.2 & 40.0 & 50.0 & 60.0 & 66.8 \\
\hline
\end{tabular}

ter the composition of biological cells (Kovacs and Keresztes, 2002). Thereupon, A. niger G-60 was mutated with ${ }^{60} \mathrm{Co}-\gamma$-rays in this study because of their strong penetration capability. Random selection and screening of mutants is a time-consuming laborious process. To reduce labour intensity, the rational selection approach was adopted basing on the selection of mutants having higher growth capability in selecting medium agar plates than the parental strain. After two days of incubation, the diameter of mutant colonies was measured and compared with the colony diameter of parental strain G-60. A single colony of mutant with a diameter $\geq 3.3 \mathrm{~cm}, \leq 2.7 \mathrm{~cm}$, or between 2.7 and $3.3 \mathrm{~cm}$ was considered a forward-, negative- or nonmutation strain, respectively.

As shown in Figure 1, the colony counts with forward-mutation (namely having enhanced enzyme-producing ability) were 5, 6 and 2 for 800, 1000 and 1200 Gy irradiation, respectively. Similarly, the colony counts with negative mutation (namely having decreased enzyme-producing ability) were 22, 8 and 33 for 800,1000 and 1200 Gy irradiation, respectively. Thus it can be seen, low to moderate doses of $\gamma$-ray irradiation (800 and $1000 \mathrm{~Gy}$ ) could produce more forward mutants relative to high dose of irradiation (1200 Gy). Both the lowest rate of forward mutation $(2.1 \%)$ and the highest rate of negative mutation (34.4\%) occurred under 1200 Gy irradiation. This indicates that high irradiation doses seriously damage cell metabolism regulation system, and cell growth deteriorates due to the loss of self-repair ability of cells during damage recovery (Agarwal et al., 2008).

To further examine inulinase productivity of mutants, six forward mutants (designated A1, C10, D6, E12, F3 and F4 respectively) and three negative mutants (designated C2, D11 and G3 respectively) were selected for enzyme activity assays. Figure 2 demonstrates inulinase activity produced by both parental strain G-60 and 9 mutants obtained with different doses of irradiation. As shown, the three negative mutants (C2, G3 and D11) had significantly lower enzyme productivity than strain G-60. The other six forward mutants exhibited higher inulinase activity than the parental one. Among them, mutant E12 possessed the maximum inulinase productivity $(128 \mathrm{U} / \mathrm{mL})$, about 2.7 times higher than that of the parent strain A. niger G-60. Thereupon, the mutant E12 was finally selected for optimization of inulinase production conditions in the following experiments.

\section{Plackett-Burman experimental design}

The Plackett-Burman experimental design for 12 trials with two levels for each variable and the corresponding inulinase production are presented in Table 3. To approach the neighborhood of the optimum response, a fitted firstorder model for inulinase production was obtained from the Plackett-Burman experimental design as follows: 


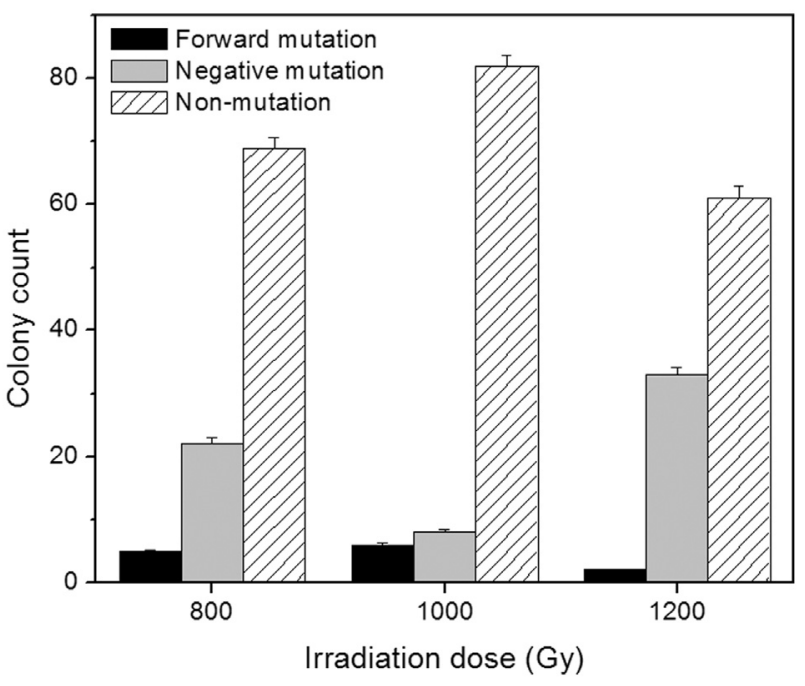

Figure 1 - Colony number of surviving cells grown in selecting medium agar plates after two days of incubation under different irradiation doses. Error bars represent the standard error associated with triplicate experiments. A single colony of mutant with a diameter $\geq 3.3 \mathrm{~cm}, \leq 2.7 \mathrm{~cm}$, or between 2.7 and $3.3 \mathrm{~cm}$ was considered a forward-, negative- or non-mutation strain, respectively.

$$
\begin{aligned}
& Y=-78.60+9.23 X_{1}+1.54 X_{2}-33.46 X_{3}+ \\
& 0.12 X_{5}+54.39 X_{6}+1.33 X_{7}-0.98 X_{9}+0.71 X_{10}
\end{aligned}
$$

The coefficient of each variable in Eq. (3) represents the influential degree of this variable on inulinase yield. The results of statistical analyses are shown in Table 1. A factor with a confidence level greater than 95\% ( $\mathrm{p} \leq 0.05)$ was considered to have a significant effect on inulinase yield and was selected for further study. The linear regression coefficient $R^{2}$ was 0.9350 and the p value was 0.0285 $(<0.05)$ for the model. These results indicate that the model was suitable for Plackett-Burman experimental design. The $\mathrm{p}$ value for peptone concentration $\left(\mathrm{X}_{6}\right)$ was less than 0.01

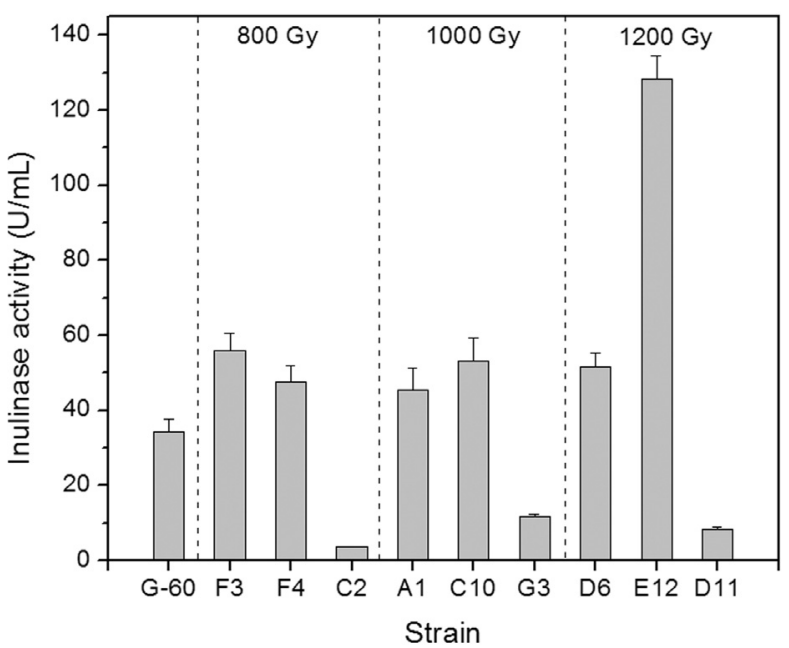

Figure 2 - Inulinase yield of parental strain G-60 and mutants after cultivation at $28{ }^{\circ} \mathrm{C}$ and $180 \mathrm{rpm}$ for $60 \mathrm{~h}$ using the fermentation medium.

with a $99 \%$ confidence, indicating that peptone concentration had an extremely significant effect on inulinase yield. The $\mathrm{p}$ values for JAP concentration $\left(\mathrm{X}_{1}\right)$ and solution volume $\left(X_{9}\right)$ were both less than 0.05 with a $95 \%$ confidence. These results clearly suggest that, in all the factors examined, peptone concentration, JAP concentration and solution volume were the most significant for inulinase production, and other factors had no significant effect on inulinase production. Peptone concentration was found to be the most influential factor, followed by solution volume and JAP concentration. Of the three significant variables identified, solution volume exerted a negative influence, while peptone concentration and JAP concentration exerted a positive influence on inulinase yield. Thus, these variables were selected as studied objects for the following experiments.

\begin{tabular}{|c|c|c|c|c|c|c|c|c|c|c|c|c|}
\hline No. & $X_{1}$ & $\mathrm{X}_{2}$ & $\mathrm{X}_{3}$ & $\mathrm{X}_{4}$ & $\mathrm{X}_{5}$ & $\mathrm{X}_{6}$ & $\mathrm{X}_{7}$ & $\mathrm{X}_{8}$ & $\mathrm{X}_{9}$ & $\mathrm{X}_{10}$ & $\mathrm{X}_{11}$ & Inulinase yield $(\mathrm{U} / \mathrm{mL})$ \\
\hline 1 & -1 & -1 & 1 & -1 & 1 & 1 & -1 & 1 & 1 & 1 & -1 & 125 \\
\hline 2 & 1 & 1 & -1 & -1 & -1 & 1 & -1 & 1 & 1 & -1 & 1 & 91.0 \\
\hline 3 & 1 & -1 & 1 & 1 & -1 & 1 & 1 & 1 & -1 & -1 & -1 & 156 \\
\hline 4 & 1 & 1 & -1 & 1 & 1 & 1 & -1 & -1 & -1 & 1 & -1 & 110 \\
\hline 5 & -1 & 1 & 1 & 1 & -1 & -1 & -1 & 1 & -1 & 1 & 1 & 77.4 \\
\hline 6 & -1 & 1 & 1 & -1 & 1 & 1 & 1 & -1 & -1 & -1 & 1 & 133 \\
\hline 7 & -1 & -1 & -1 & 1 & -1 & 1 & 1 & -1 & 1 & 1 & 1 & 93.3 \\
\hline 8 & -1 & 1 & -1 & 1 & 1 & -1 & 1 & 1 & 1 & -1 & -1 & 122 \\
\hline 9 & -1 & -1 & -1 & -1 & -1 & -1 & -1 & -1 & -1 & -1 & -1 & 107 \\
\hline 10 & 1 & -1 & -1 & 1 & 1 & -1 & -1 & -1 & 1 & -1 & -1 & 104 \\
\hline 11 & 1 & -1 & -1 & -1 & 1 & -1 & -1 & 1 & -1 & 1 & 1 & 115 \\
\hline 12 & 1 & 1 & 1 & -1 & -1 & -1 & 1 & -1 & 1 & 1 & -1 & 126 \\
\hline
\end{tabular}

Table 3 - The design and results of Plackett-Burman design. 


\section{The path of steepest ascent}

The above results indicate that compared with other factors, peptone concentration, JAP concentration, and solution volume can significantly influence inulinase yield. Moreover, Eq. (3) shows that the coefficient of $X_{9}$ was negative while the coefficients of $\mathrm{X}_{1}$ and $\mathrm{X}_{6}$ were positive, which means that the decrease in solution volume and the increase in concentrations of JAP and peptone can exert positive effect on inulinase production. The path of the steepest ascent was used to search for the proper direction to change the levels of these three factors while keeping the other factors constant at the levels found in basal fermentation medium. The experimental design and corresponding results are listed in Table 4 . The results indicate that the yield of inulinase was maximum when the concentrations of JAP and peptone, and solution volume were selected to be $70.0 \mathrm{~g} / \mathrm{L}, 22.5 \mathrm{~g} / \mathrm{L}$ and $50.0 \mathrm{~mL}$, respectively. These results suggest that this level for each of the three factors was near the region of maximum inulinase production response.

\section{Central composite design and response surface methodology}

To further enhance inulinase production by the mutant E12, central composite design and the response surface methodology were employed to analyze the interactive effect of JAP concentration, peptone concentration and solution volume and to obtain an optimum for the inulinase production by the mutant E12. The values of the three variables in the run 3 obtained from the steepest ascent path (Table 4) were taken as the center points with the other variables fixed at low level (Table 1). The design and results of the experiments carried out with the central composite design are listed in Table 5.

The analysis of variance (ANOVA) was employed for the determination of the significant parameters. The results obtained were submitted to ANOVA on Design-Expert 8.0 and the regression model was given as the following equation:

$$
\begin{aligned}
& Y=-3650.52+55.28 A+4246 B+54.08 C- \\
& 0.45 A^{2}-1.01 B^{2}-0.55 C^{2}+0.29 A B- \\
& 0.12 A C-036 B C
\end{aligned}
$$

Table 4 - The designs and results of path of steepest ascent.

\begin{tabular}{lcccc}
\hline No. & JAP $(\mathrm{g} / \mathrm{L})$ & Peptone $(\mathrm{g} / \mathrm{L})$ & $\begin{array}{c}\text { Solution } \\
\text { volume }(\mathrm{mL})\end{array}$ & $\begin{array}{c}\text { Inulinase yield } \\
(\mathrm{U} / \mathrm{mL})\end{array}$ \\
\hline 1 & 50.0 & 12.5 & 60 & 97.0 \\
2 & 60.0 & 17.5 & 55 & 138 \\
3 & 70.0 & 22.5 & 50 & 166 \\
4 & 80.0 & 27.5 & 45 & 120 \\
5 & 90.0 & 32.5 & 40 & 104 \\
6 & 100.0 & 37.5 & 35 & 81.7 \\
\hline
\end{tabular}

where $Y$ is inulinase activity, $A$ is JAP concentration, $B$ is peptone concentration, $C$ is solution volume (Table 2). The ANOVA of the quadratic regression model demonstrates that Eq. (4) is a highly significant model, as is evident from the Fisher's $F$-test with a very low probability value ( $F$ value $=7.70)($ Table 6$)$. The p value $(0.0013)$ was less than 0.01 with a $99 \%$ confidence (Table 6 ), indicating model terms were extremely significant. Of the first terms $(A, B$, $C)$, JAP concentration $(A)$ had significant effect on inulinase yield $(\mathrm{p}<0.05)$. Of the quadratic terms $\left(A^{2}, B^{2}, C^{2}\right)$, concentrations of JAP and peptone and solution volume had extremely significant effect on inulinase yield $(\mathrm{p}<0.01)$. However, the interaction terms $(A B, A C, B C)$ had no significant effect on inulinase yield $(\mathrm{p}>0.05)$.

The interactions of the three components and their optimum level in inulinase production were further analyzed through response surface methodology. The three-dimension response surface curves and respective contour plots are presented in Figures 3-5. Three-dimensional graphs were generated for the combination of the two variables, while keeping the other one at their optimum levels determined by the path of steepest ascent for inulinase production by the mutant E12. It can be seen that the response surface was convex, suggesting that the optimum conditions are well-defined and there existed a maximum for each variable. According to the results of the response surface analysis, the predicted maximum production of inuli-

Table 5 - The designs and results of central composite design.

\begin{tabular}{lcccc}
\hline No. & JAP (A) & Peptone (B) & $\begin{array}{c}\text { Solution } \\
\text { volume }(\mathrm{C})\end{array}$ & $\begin{array}{c}\text { Inulinase yield } \\
(\mathrm{U} / \mathrm{mL})\end{array}$ \\
\hline 1 & 1 & -1 & 1 & 151 \\
2 & 0 & 0 & 0 & 166 \\
3 & 0 & 0 & 0 & 166 \\
4 & 1.68 & 0 & 0 & 118 \\
5 & 0 & -1.68 & 0 & 131 \\
6 & 0 & 0 & 0 & 166 \\
7 & -1 & -1 & -1 & 81.1 \\
8 & 1 & -1 & -1 & 137 \\
9 & -1 & 1 & -1 & 117 \\
10 & 0 & 0 & 0 & 166 \\
11 & 0 & 0 & -1.68 & 113 \\
12 & -1.68 & 0 & 0 & 152 \\
13 & 1 & 1 & 1 & 109 \\
14 & 0 & 0 & 0 & 166 \\
15 & -1 & -1 & 1 & 144 \\
16 & 0 & 1.68 & 0 & 137 \\
17 & 0 & 0 & 0 & 166 \\
18 & 1 & 1 & -1 & 138 \\
19 & 0 & 0 & 1.68 & 127 \\
20 & -1 & 1 & 1 & 132 \\
\hline & & & &
\end{tabular}


nase was $193 \mathrm{U} / \mathrm{mL}$ when the concentrations of JAP and peptone, and solution volume were $66.5 \mathrm{~g} / \mathrm{L}, 29.1 \mathrm{~g} / \mathrm{L}$ and
$49.4 \mathrm{~mL}$, respectively, and the rest of the variables were kept at zero level (Table 2). In the present study, inorganic

Table 6 - Analysis of variance (ANOVA) for regression of central composite design.

\begin{tabular}{lcc}
\hline Variance origin & $F$ value & p value \\
\hline $\mathrm{A}$ & 6.97 & $0.0247^{*}$ \\
$\mathrm{~B}$ & 0.094 & 0.8295 \\
$\mathrm{C}$ & 0.74 & 0.4099 \\
$\mathrm{~A}^{2}$ & 16.55 & $0.0023^{* *}$ \\
$\mathrm{~B}^{2}$ & 26.17 & $0.0005^{* *}$ \\
$\mathrm{C}^{2}$ & 24.34 & $0.0006^{* *}$ \\
$\mathrm{AB}$ & 2.18 & 0.1708 \\
$\mathrm{AC}$ & 0.041 & 0.8434 \\
\hline
\end{tabular}

\begin{tabular}{lcc}
\hline Variance origin & $F$ value & $\mathrm{p}$ value \\
\hline $\mathrm{BC}$ & 3.20 & 0.1040 \\
Model & 7.70 & $0.0013^{* *}$ \\
First term & 0.58 & 0.6375 \\
Quadratic term & 18.71 & 0.0002 \\
Interaction term & 0.35 & 0.7864 \\
Lack of fit & 2.22 & 0.2007
\end{tabular}

*Significant at $95 \%$ confidence degree $(\mathrm{p}<0.05) . * *$ Extremely significant level at $99 \%$ confidence degree $(\mathrm{p}<0.01)$.
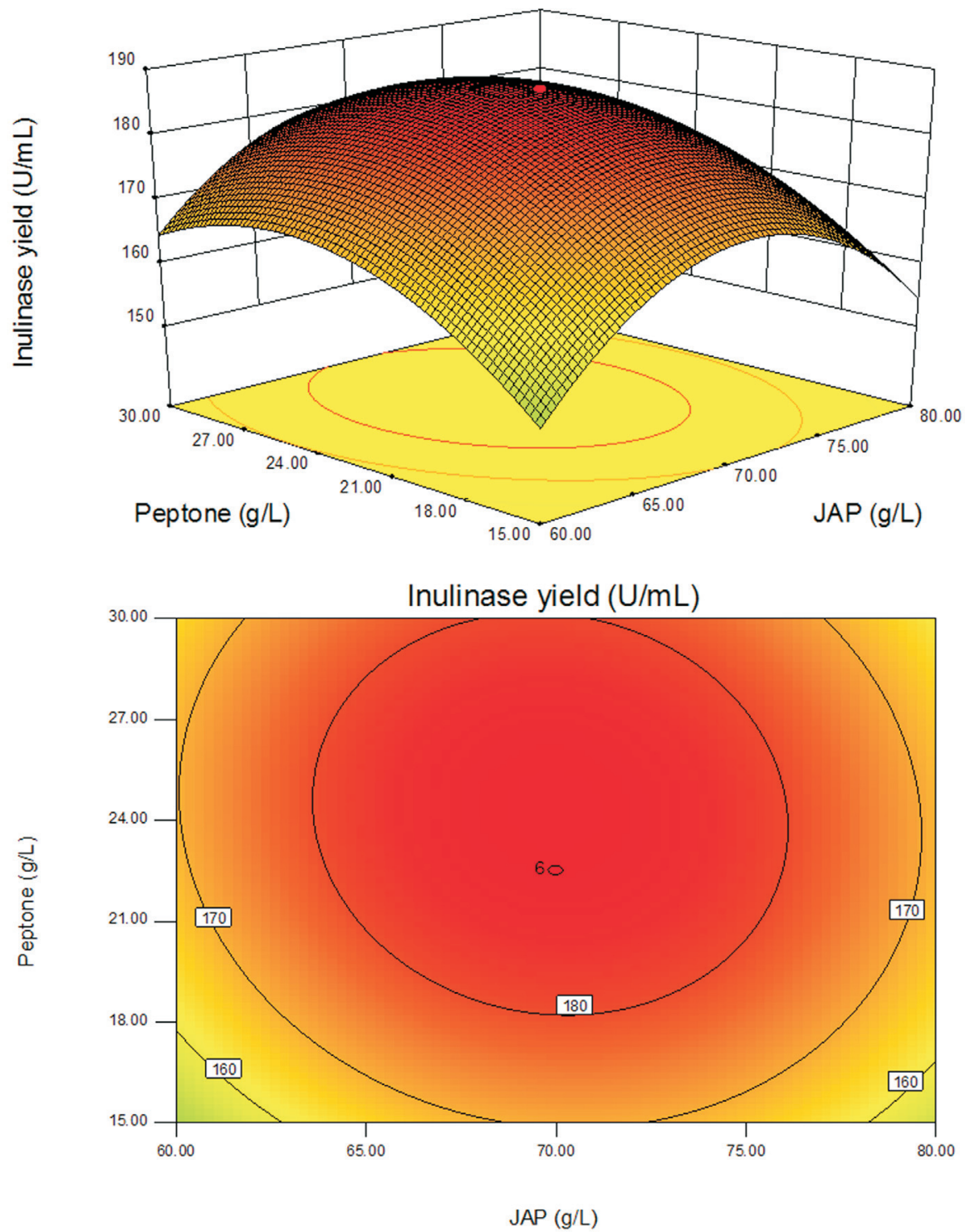

Figure 3 - The response surface plot and the corresponding contour plot showing the effects of JAP and peptone on inulinase production by mutant E12. Solution volume level, $50.0 \mathrm{~mL}$. 

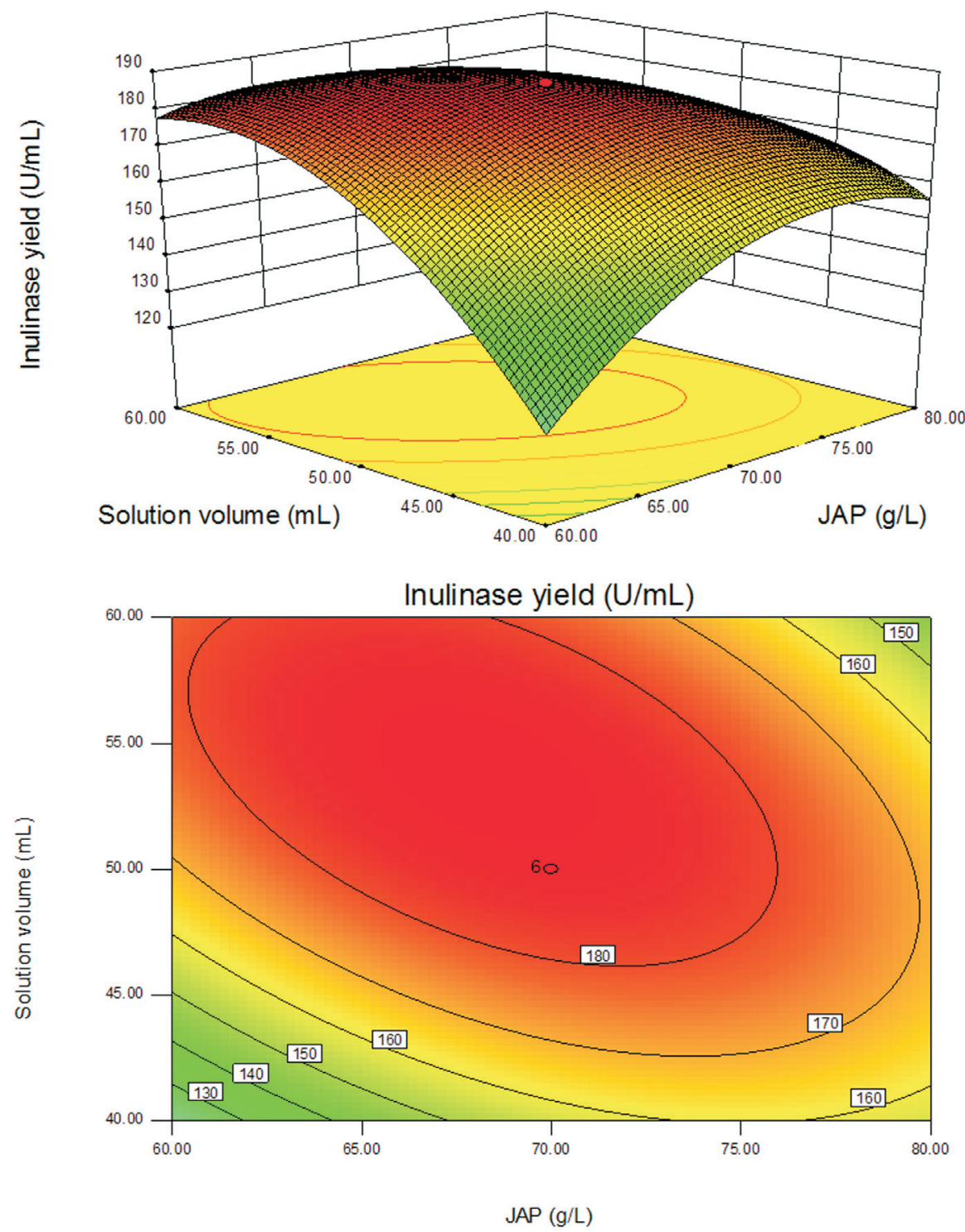

Figure 4 - The response surface plot and the corresponding contour plot showing the effects of JAP and solution volume on inulinase production by mutant E12. Peptone level, $22.5 \mathrm{~g} / \mathrm{L}$.

salts such as ammonium, magnesium and sulfate ions were not included in the fementation medium composition. This is because the carbon source used (JAP) is a crude material containing a variety of inorganic nutrients.

The mutant E12 was cultured with the optimized medium to examine the effectiveness of the model equations in successfully predicting the optimum response values. The set of conditions predicted by response surface methodology as optimum was used for experimental validation. The inulinase yield obtained from experiments using the mutant E12 culture was $185 \mathrm{U} / \mathrm{mL}$, a value that was similar to that predicted by response surface methodology model $(193 \mathrm{U} / \mathrm{mL})$, indicating that the model can be indeed employed for optimizing culture conditions for inulinase production. Thus, the yield of inulinase using the optimized culture medium was approximately 1.4 -fold of that obtained when basal culture medium was used.

Optimal medium composition is vital for increasing inulinase production using submerged cultures. Statistical experimental design provides a systematic and efficient means of realizing desired goals, and it helps in understanding the interactions among the process parameters at varying levels and in calculating an optimaum level of each variable for the maximum product yield (Trivedi et al., 2012). Many previous studies have demonstrated that inulinase production by Aspergillus species could be remarkably enhanced by the optimization of cultivation conditions and medium compositions (Skowronek and Fiedurek, 2004; Kumar et al., 2005; Trivedi et al., 2012; Abd El Aty et al., 2014). 

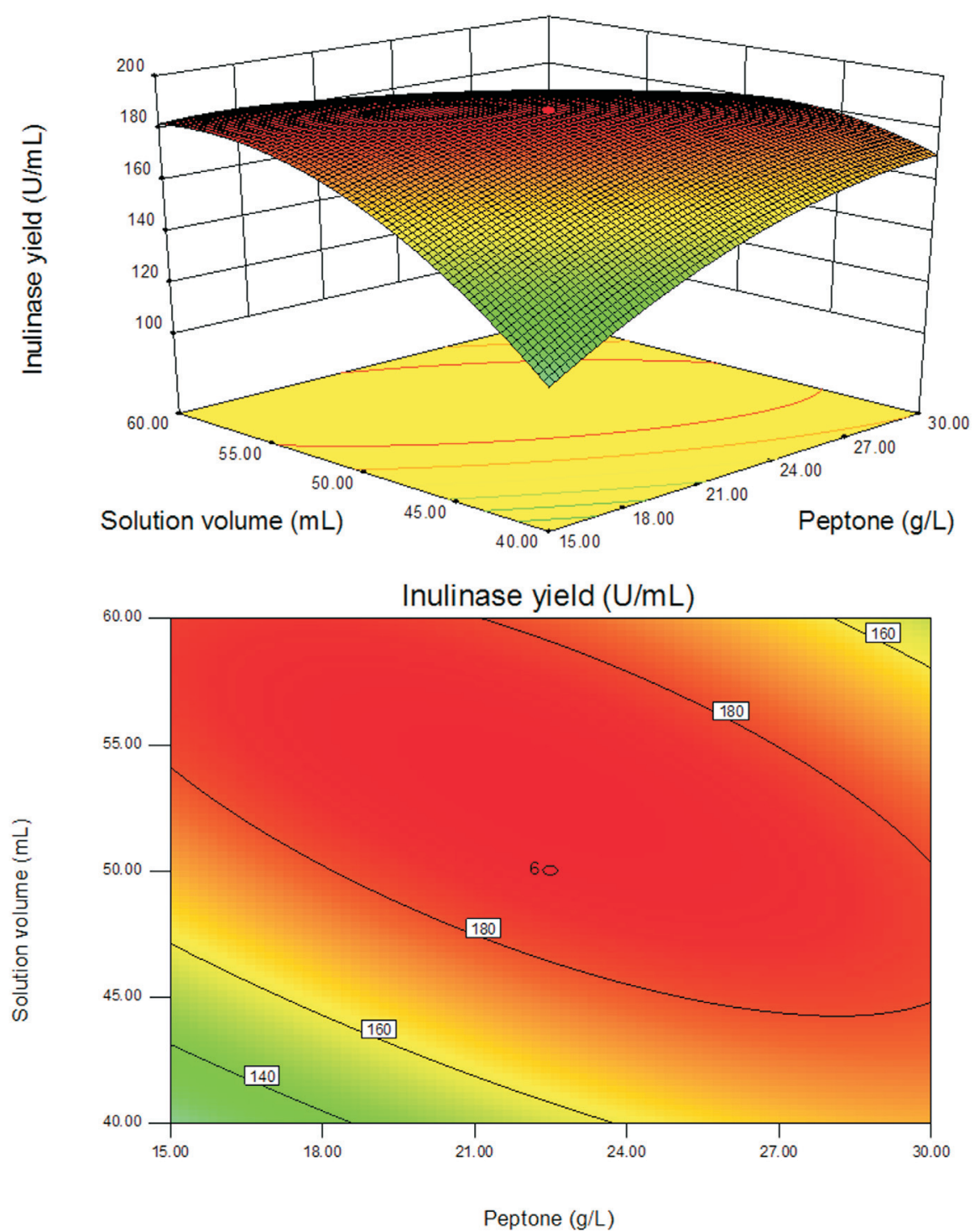

Figure 5 - The response surface plot and the corresponding contour plot showing the effects of peptone and solution volume on inulinase production by mutant E12. JAP level, $70.0 \mathrm{~g} / \mathrm{L}$.

In this study, the mutant E12 showed higher inulinase production ability than some other Aspergillus species. For example, the highest extracellular enzyme activity of $A$. niger 13/36 (Skowronek and Fiedurek, 2004) and A. niger AUP19 (Kumar et al., 2005) was found to be 80 and 176 $\mathrm{U} / \mathrm{mL}$, respectively. Moreover, the mutant E12 had a lower nutrient demand than strains $13 / 36$ and AUP19, as they needed sucrose or galactose as additional carbon source.

\section{Conclusions}

Results from this study have demonstrated that $A$. niger G-60 was an efficient inulinase producer. Also, it was effective to enhance inulinase yield of the strain by nuclear irradiation mutation. A mutant strain of A. niger G-60 (designated as E12), with inulinase yield improved more than 2.7-fold compared to the parental strain, was obtained by ${ }^{60} \mathrm{Co}-\gamma$ mutagenesis treatment in this study. The application of response surface methodology design resulted in an improvement of inulinase yield. The mutant E12 could produce inulinase at a high level and therefore had significant commercial potential. Further research is needed to explore the transformed functional genes and regulated metabolic network, which are related to inulinase porduction in $A$. niger G-60 cells mutated by nuclear irradiation.

\section{Acknowledgments}

This study has been funded by the opening project of the State Key Laboratory of Bioreactor Engineering in the year 2011 (No. SKLBE-KF-019). 


\section{References}

Abd El Aty AA, Wehaidy HR, Mostafa FA (2014) Optimization of inulinase production from low cost substrates using Plackett-Burman and Taguchi methods. Carbohyd Polym 102:261-268.

Agarwal R, Rane SS, Sainis, JK (2008) Effects of ${ }^{60} \mathrm{Co} \gamma$ radiation on thylakoid membrane functions in Anacystis nidulans. J Photochem Photobiol B 91:9-19.

Cheng J, Feng J, Sun J et al. (2014) Enhancing the lipid content of the diatom Nitzschia sp. by ${ }^{60} \mathrm{Co}-\gamma$ irradiation mutation and high-salinity domestication. Energy DOI: 10.1016/j.energy.2014.06.009.

Feng J, Cheng J, Cheng R et al. (2014) Screening the diatom Nitzschia sp. re-mutated by ${ }^{137} \mathrm{Cs}-\gamma$ irradiation and optimizing growth conditions to increase lipid productivity. J Appl Phycol DOI:10.1007/s10811-014-0367-6.

Gao W, Bao Y, Liu Y et al. (2009) Characterization of thermostable endoinulinase from a new strain Bacillus Smithii T7. Appl Biochem Biotechnol 157:498-506.

Ge XY, Qian H, Zhang WG (2009) Improvement of L-lactic acid production from Jerusalem artichoke tubers by mixed culture of Aspergillus niger and Lactobacillus sp. Bioresour Technol 100:1872-1874.

Gill PK, Manhas RK, Singh P (2006) Hydrolysis of inulin by immobilized thermostable extracellular exoinulinase from Aspergillus fumigatus. J Food Eng 76:369-375.

Hu N, Yuan B, Sun J et al. (2012) Thermotolerant Kluyveromyces marxianus and Saccharomyces cerevisiae strains representing potentials for bioethanol production from Jerusalem artichoke by consolidated bioprocessing. Appl Microbiol Biotechnol 95:1359-1368.

Kovacs E, Keresztes A (2002) Effect of gamma and UV-B/C radiation on plant cells. Micron 33:199-210.

Kumar GP, Kunamneni A, Prabhakar T et al. (2005) Optimization of process parameters for the production of inulinase from a newly isolated Aspergillus niger AUP19. World J Microbiol Biotechnol 21:1359-1361.

Li Y, Liu GL, Chi ZM (2013) Ethanol production from inulin and unsterilized meal of Jerusalem artichoke tubers by Saccharomyces sp. W0 expressing the endo-inulinase gene from Arthrobacter sp. Bioresour Technol 147:254-259.

Maren AK (2002) Identification of Common Aspergillus Species. Central Bureau Voor Schimmel Cultures Publishers, pp. 1-62.

Peterson SW (2008) Phylogenetic analysis of Aspergillus species using DNA sequences from four loci. Mycologia 100:205226.
Reddy LVA, Wee YJ, Yun JS et al. (2008) Optimization of alkaline protease production by batch culture of Bacillus sp. RKY3 through Plackett-Burman and response surface methodological approaches. Bioresour Technol 99:2242-2249.

Saengthongpinit W, Sajjaanantakul T (2005) Influence of harvest time and storage temperature on characteristics of inulin from Jerusalem artichoke (Helianthus tuberosus L.) tubers. Postharvest Boil Technol 37:93-100.

Sheng J, Chi Z, Li J et al. (2007) Inulinase production by the marine yeast Cryptococcus aureus $\mathrm{G} 7 \mathrm{a}$ and inulin hydrolysis by the crude inulinase. Process Biochem 42:805-811.

Silva DM, Batista LR, Rezende EF et al. (2011) Identification of fungi of the genus Aspergillus section nigri using polyphasic taxonomy. Brazil J Microbiol 42:761-773.

Sirisansaneeyakul S, Worawuthiyanan N, Vanichsriratana W et al. (2006) Production of fructose from inulin using mixed inulinases from Aspergillus niger and Candida guilliermondii. World J Microbiol Biotechnol 23:543-552.

Skowronek M, Fiedurek J (2004) Optimisation of inulinase production by Aspergillus niger using simplex and classical method. Food Technol Biotechnol 42:141-146.

Trivedi S, Divecha J, Shah A (2012) Optimization of inulinase production by a newly isolated Aspergillus tubingensis CR16 using low cost substrates. Carbohyd Polym 90:483490.

Wang L, Xue Z, Zhao B et al. (2013) Jerusalem artichoke powder: A useful material in producing high-optical-purity 1-lactate using an efficient sugar-utilizing thermophilic Bacillus coagulans strain. Bioresour Technol 130:174-180.

Xie H, Zhou Y, Hu J et al. (2014) Production of astaxanthin by a mutant strain of Phaffia rhodozyma and optimization of culture conditions using response surface methodology. Ann Microbiol DOI:10.1007/s13213-013-0790-y.

Yuan WJ, Zhao XQ, Ge XM et al. (2008) Ethanol fermentation with Kluyveromyces marxianus from Jerusalem artichoke grown in salina and irrigated with a mixture of seawater and freshwater. J Appl Microbiol 105:2076-2083.

Zhang L, Zhao C, Zhu D et al. (2004) Purification and characterization of inulinase from Aspergillus niger AF10 expressed in Pichia pastoris. Protein Express Purif 35:272-275.

Zhang T, Chi Z, Zhao CH et al. (2010) Bioethanol production from hydrolysates of inulin and the tuber meal of Jerusalem artichoke by Saccharomyces sp. W0. Bioresour Technol 101:8166-8170.

Associate Editor: Gisele Monteiro de Souza

All the content of the journal, except where otherwise noted, is licensed under a Creative Commons License CC BY-NC. 